San Jose State University

SJSU ScholarWorks

Master's Theses

Master's Theses and Graduate Research

1994

\title{
Is linear displacement information or angular displacement information used during the adaptation of pointing responses to an optically shifted image?
}

Abigail B. Bautista

San Jose State University

Follow this and additional works at: https://scholarworks.sjsu.edu/etd_theses

\section{Recommended Citation}

Bautista, Abigail B., "Is linear displacement information or angular displacement information used during the adaptation of pointing responses to an optically shifted image ?" (1994). Master's Theses. 895. DOI: https://doi.org/10.31979/etd.gmnz-6qsp

https://scholarworks.sjsu.edu/etd_theses/895

This Thesis is brought to you for free and open access by the Master's Theses and Graduate Research at SJSU ScholarWorks. It has been accepted for inclusion in Master's Theses by an authorized administrator of SJSU ScholarWorks. For more information, please contact scholarworks@sjsu.edu. 


\section{INFORMATION TO USERS}

This manuscript has been reproduced from the microfilm master. UMI films the text directly from the original or copy submitted. Thus, some thesis and dissertation copies are in typewriter face, while others may be from any type of computer printer.

The quality of this reproduction is dependent upon the quality of the copy subraitted. Broken or indistinct print, colored or poor quality illustrations and photographs, print bleedthrough, substandard margins, and improper alignment can adversely affect reproduction.

In the unlikely. event that the author did not send UMI a complete manuscript and there are missing pages, these will be noted. Also, if unauthorized copyright material had to be removed, a note will indicate the deletion.

Oversize materials (e.g., maps, drawings, charts) are reproduced by sectioning the original, beginning at the upper left-hand corner and contimuing from left to right in equal sections with small overlaps. Each original is also photographed in one exposure and is included in reduced form at the back of the book.

Photographs included in the original manuscript have been reproduced xerographically in this copy. Higher quality $6^{\prime \prime} \times 9^{n}$ black and white photographic prints are available for any photographs or illustrations appearing in this copy for an additional charge. Contact UMI directly to order.

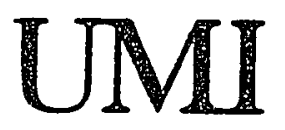

A Bell \& Howell information Company 
$\cdots$

- 
IS LINEAR DISPLACEMENT INFORMATION OR ANGULAR DISPLACEMENT INFORMATION USED DURING THE ADAPTATION OF POINTING RESPONSES TO AN OPTICALLY SHIFTED IMAGE?

\author{
A Thesis \\ Presented to \\ the Faculty of the Department of Psychology of \\ San Jose State University
}

In Partial Fulfillment of the Requirements of the Degree

Master of Arts

by

Abigail B. Bautista

August, 1994 
UMI Number: 1361153

JMI Microform Edition 1361153

Copyright 1995, by UMI Company. All rights reserved.

This microform edition is protected against unauthorized copying under Title 17, United States Code.

\section{UMI \\ 300 North Zeeb Road \\ Ann Arbor, MI 48103}


(C) 1994

Abigail B. Bautista

ALL RIGHTS RESERVED 
APPROVED FOR THE DEPARTMENT OF PSYCHOLOGY

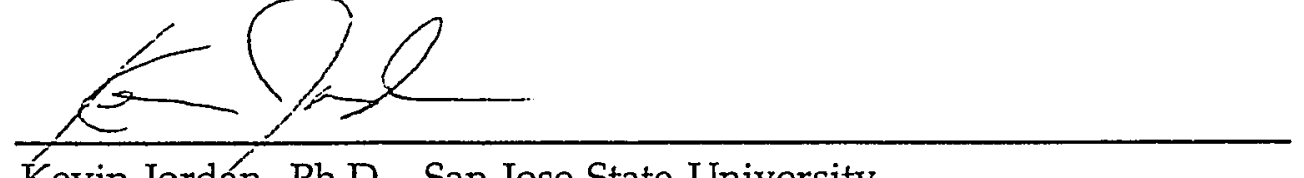

Kevin Jordán Ph.D., San Jose State University,

Thesis Chairperson

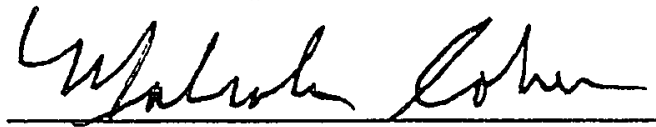

Malcolm Cohen Ph.D., NASA Ames Research Center,

Thesis Committee Member

$\frac{\text { Roller } \mathrm{C} \text { Cepren }}{\text { Robert Cooper Ph.D., San Jose State University, }}$

Thesis Committee Member

APPROVED FOR THE UNIVERSITY

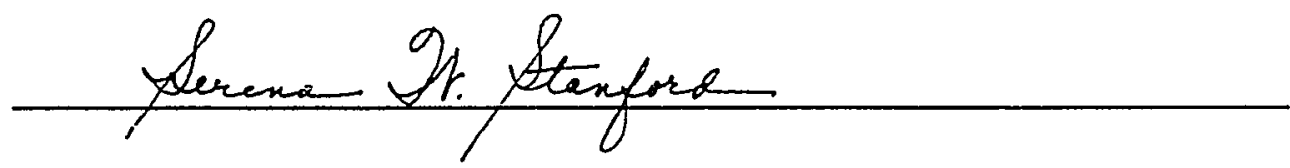




\begin{abstract}
IS LINEAR DISPLACEMENT INFORMATION OR ANGULAR DISPLACEMENT INFORMATION USED DURING THE ADAPTATION OF POINTING RESPONSES TO AN OPTICALLY SHIFTED IMAGE?
\end{abstract}

by Abigail B. Bautista

Twenty-four observers looked through a pair of 20 diopter wedge prisms and pointed to an image of a target which was displaced vertically from eye level by $6 \mathrm{~cm}$ at a distance of $30 \mathrm{~cm}$. Observers pointed 40 times, using only their right hand, and received error-corrective feedback upon termination of each pointing response (terminal visual feedback). At three testing distances, 20,30, and $40 \mathrm{~cm}$, ten pre-exposure and ten post-exposure pointing responses were recorded for each hand, as observers reached to a mirror-viewed target located at eye level. The difference between pre- and post-exposure pointing response (adaptive shift) was compared for both Exposed and Unexposed hands across all three testing distances. The data were assessed according to the results predicted by two alternative models for processing spatial-information: one using angular displacement information and another using linear displacement information. The angular model of spatial location mapping best predicted the observer's pointing response for the Exposed hand; linear adaptive shift increased as a function of the testing distance suggesting that observers encoded target location using angular information. 


\section{ACKNOWLEDGEMENTS}

This study was funded by NASA grant UPN-199-16-12-08 to Dr. Malcolm M. Cohen at NASA Ames Research Center.

I would like to express my appreciation to all those who have guided me through the process of writing this thesis. I thank Professor Kevin Jordan and Dr. Malcolm M. Cohen for their pedagogy in experimental design and the hours they invested showing me what "strong inference" means. I thank Dr. Robert Cooper for his insight and I thank Howard Tokunaga for guiding me down the path of ANOVA.

Special thanks to the subjects who displayed wonderful and cooperative spirit. Lastly, I thank all my family and friends whose support made all things possible. This thesis is dedicated to my father Jose C. Bautista. 


\section{TABLE OF CONTENTS}

SECTION

PAGE

INTRODUCTION 3

Information System Models $\quad 6$

Linear Encoding $\quad 6$

$\begin{array}{ll}\text { Angular Encoding } & 7\end{array}$

$\begin{array}{ll}\text { Apparatus } & 7\end{array}$

$\begin{array}{ll}\text { Motor Task Decrement } & 7\end{array}$

Local vs Central System Representation $\quad 8$

Proposed Experiment $\quad 9$

$\begin{array}{ll}\text { METHOD } & 9\end{array}$

$\begin{array}{ll}\text { Research Participants } & 9\end{array}$

$\begin{array}{ll}\text { Apparatus } & 10\end{array}$

$\begin{array}{ll}\text { Procedures } & 10\end{array}$

Data Scoring 13

$\begin{array}{ll}\text { RESULTS } & 14\end{array}$

Pre- and Post-exposure Pointing Responses 14

Adaptive Shift Trials 1-5 19

$\begin{array}{ll}\text { Research Participants } & 9\end{array}$

$\begin{array}{ll}\text { Apparatus } & 10\end{array}$

DISCUSSION 22

Exposed vs Unexposed $\quad 22$

Intermanual Transfer 22

Radial Model 24

Further Reasearch 24

$\begin{array}{ll}\text { REFERENCES } & 27\end{array}$ 


\section{LIST OF TABLES}

TABLE

PAGE

1. Means and Standard Deviations for Pre-exposure and Post-exposure Pointing Responses Collapsed Across Conditions of Target Position and Test Hand.

2. Means and Standard Deviations for the Difference Between Pre-exposure and Post-exposure Pointing Responses Collapsed Across Target Position.

3. Mean Adaptive Shift for Trials 1-5 for the Exposed and Unexposed Hands Over the Three Testing Differences

Collapsed Across Trials 1-5 and Direction of Target Shift. 


\section{LIST OF FIGURES}

FIGURE

PAGE

1. The hand-eye coordination apparatus during a training

phase with the target shifted downward (prism base up). 11

2. The effect of Trials on Pre- and Post-exposure Pointing

Responses averaged Over Test Hand, Test Distance,

and Target Position.

18

3. Linear relationship between adaptive shift and

Test Distance for the Exposed Hand. 
Is Linear Displacement Information or Angular Displacement Information Used During the Adaptation of Pointing Responses to an Optically Shifted Image?

\author{
Abigail B. Bautista
}

San Jose State University

Running head: INFORMATION USED TO ENCODE LOCATION IN SPACE

\title{
Footnotes
}

Requests for reprints should be sent to Abigail B. Bautista, Department of Psychology, San Jose State University, San Jose, California 95192. 


\begin{abstract}
Twenty-four observers looked through a pair of 20 diopter wedge prisms and pointed to an image of a target which was displaced vertically from eye level by $6 \mathrm{~cm}$ at a distance of $30 \mathrm{~cm}$. Observers pointed 40 times, using only their right hand, and received error-corrective feedback upon termination of each pointing response (terminal visual feedback). At three testing distances, 20,30, and $40 \mathrm{~cm}$, ten pre-exposure and ten post-exposure pointing responses were recorded for each hand, as observers reached to a mirror-viewed target located at eye level. The difference between pre- and post-exposure pointing response (adaptive shift) was compared for both Exposed and Unexposed hands across all three testing distances. The data were assessed according to the results predicted by two alternative models for processing spatial-information: one using angular displacement information and another using linear displacement information. The angular model of spatial location mapping best predicted the observer's pointing response for the Exposed hand; linear adaptive shift increased as a function of the testing distance suggesting that observers encoded target location using angular information.
\end{abstract}


Is Linear Displacement Information or Angular Displacement Information Used During the Adaptation of Pointing Responses to an Optically Shifted Image?

Daily actions that we rarely think about such as reaching for a cup of coffee or inputting a number on a telephone keypad are common uses of hand-eye coordination. These routine movements are accomplished with little attention to how our hand achieves its goal. Mundane reaching tasks suddenly become challenging when we don a new pair of corrective lenses; our environment is altered by the new relationships between our changed visual world and the unchanged objective space surrounding us. Optical distortions along the edges of the new lenses will produce either a compression or expansion of the visual input information; thus, reaching quickly for an object will result in error.

Errors that occur when reaching to an object that is optically shifted quickly disappear as the observer receives feedback. Since the physical location of the object has not changed, a new relationship develops between the perceived and the physical location of that stimulus. With minimal practice, typically twenty to thirty responses (Welch, 1978), the individual will again be able to execute responses accurately within that environment.

\section{Definition of Adaptation}

Adaptation to a specific physical environment can be viewed as a change in behavior that reduces discrepancies among sensory modalities (Welch, 1978). Wallace and Redding (1988, 1992a) apply this concept to visual-proprioceptive localization, suggesting that adaptation is the ability of the perceptual-motor system to maintain a spatial agreement among the 
maps of numerous coordinate subsystems (e.g. eye-head, head-neck, and arm-hand; Templeton, Howard, \& Wilkinson, 1974). They conceptualize each of these subsystems as capable of operating independently by using each of the subsystems' own sensory and effector capacities.

Overall coordinated movement is achieved through cross-talk, or translating coordinate information across subsystems. According to this theory, eye-hand coordination not only requires the mapping of visual space onto proprioceptive space, but it also demands the creation of individual maps for each of the subsystems in the hierarchy from the eye to the hand (Templeton et al., 1974). Ego-centric space is abstract; it exists as a central representation which maps space for the motor system and must be calibrated by other sensory information (Jeannerod, 1983).

Error-corrective feedback gives an individual the information needed to recalibrate this internal representation of space and reconcile any disagreements between the seen position of an object and the felt position of that same object (Held \& Freeman, 1963). This semi-permanent change allows the individual to generate appropriate efferent signals when the viewing conditions change. The newly developed relationship will persist until another disagreement between visual and motor mappings of space occurs.

Studies of adaptation to an optically-shifted target image or environment through the use of prisms have been plentiful (Cohen, 1967, 1973; Hay \& Pick, 1966; Welch, Choe, \& Heinrich, 1974). More recent work, such as Redding and Wallace (1988, 1992b) and Welch et al. (1974), has attempted to draw out the underlying visual and proprioceptive components 
of the phenomenon of prism adaptation. According to most recent models (Wallace \& Redding, 1992b; Welch et al., 1974), the various components of adaptation, such as visual adaptation and proprioceptive adaptation, are additive and can be measured as separate components. These different components of adaptation are produced to a greater or lesser extent according to the type of feedback information available during prismatic exposure (Cohen, 1967, 1973; Wallace \& Redding, 1988). When terminal feedback is given during exposure, the visual component of adaptation is of greater magnitude; if continuous feedback is given, the proprioceptive component will be of greater relative magnitude. In addition, terminal feedback will result in greater amounts of intermanual transfer or transfer of adaptation from the exposed to the unexposed hand (Cohen, 1967, 1973). Adaptation measured in the unexposed hand represents a central adaptation of eye-hand coordination. Proprioceptive (local) adaptation present in the exposed hand is theoretically absent in measures of adaptation in the unexposed hand.

In experimental paradigms in which a target's visual image is displaced optically using prisms and there is no visual feedback during arm movements, the observer will make pointing errors in the direction of the displaced image. With practice and error-corrective feedback upon the termination of the response, the observer will quickly begin to point accurately to the target. Once the prisms are removed, the observer, when pointing to the same target and without feedback, will make errors in the direction opposite the original optical displacement until feedback is given. This residual pointing error is identified throughout the literature as 
"aftereffect" (Cohen, 1973) or "negative aftereffect" (Welch, 1978), and is a common measure of adaptation by the eye-hand system.

If this resulting adaptation is a change in the visual and proprioceptive mapping of space, what kind of displacement information is being used and conserved by these systems? Is the observer reducing error by recalibration and shifting the axis of a Cartesian coordinate system? Or, does the observer shift the poles of a radial coordinate system a constant angular value? To make accurate goal-directed movements (e.g., quickly reaching for a pencil), the human motor system requires a precise coordinate system, an internal mapping of space that is referenced from the body's center (Jeannerod, 1983). Further, this system must be able to apply the variety of in-coming and outgoing information processed by the body so that when the individual sees an object, the proper motor command can be sent to intercept the object. Information System Models

There are two distinct types of information that can be used to model a spatial mapping system, one that encodes linear displacement (a Cartesian map) and another that encodes angular displacement (a radial map). If the location of a target is encoded using linear displacement information, then a constant linear adaptive shift would be recorded at any distance at which a target is placed. In contrast, if the location of a target is encoded using angular displacement information, adaptation to the image of an optically shifted target will result in a linear adaptive shift that increases as a function of increases in a target's distance.

Linear Encoding. An observer who uses linear displacement information to correct pointing errors to an optically shifted target will make 
identical compensations in all post-exposure pointing responses. This correction can be viewed as an adjustment or recalibration to the new visuospatial relationship, a shifted axis in a Cartesian coordinate system. Once the optical displacement in the environment is removed, there should be no effect due to distance. The target once again lies in the plane of the observer's eye level and all subsequent pointing responses should then lie in a plane parallel to the eye level plane.

Angular Encoding. Conversely, the coding of spatial surroundings, with regards to the movement of the performer, would best be described in a coordinate system encoded using radial directions (Rock, 1966). This egocentric representation system is simple and efficient to use when interacting with the surrounding environment. The head rotates with respect to the body and the arms and legs extend to form a sphere of interaction that is limited by the length of these limbs. Thus, a pointing response to the apparent location of a target would best be represented as a ray emanating from the center of the observer's body to that target.

An observer who uses angular displacement information to correct error will generate compensations that are dependent on target distance. The size of compensation for closer targets would be smaller and the size for farther targets would be larger than for the middle distance target. All pointing responses would lie on a plane that is rotated about an axis located along the observer's body. Motor Task Decrement

The existence of constant offset, either linear or angular, would not necessitate a change in spatial mapping and may instead reflect the use of a 
cognitive strategy to reduce error. Applied as a constant-magnitude errorcorrection, this learned motor program would be used to reduce the difference between the target image and the erroneous pointing response seen during exposure to the optically displaced environment. If generalized to all pointing responses, it implies that we learn very specific error-corrective responses that may be independent of perceptual changes.

Any decrements in the constant offset, linear or angular, for both the far and near target distances can be explained through a generalization gradient for motor tasks. Tasks practiced at one distance and ensuing performance of the same task, but with alterations of a single parameter such as distance, will result in only partial and not complete transfer of learning (Osgood, 1949; Schmidt, 1988).

\section{Local vs Central System Representation}

The pattern of negative aftereffect magnitude as tested over three testing distances using either the exposed or unexposed hand should define the type of information used within a local system and a central system of representation. Local system adaptation will be defined by the differences in response of the exposed hand and the unexposed hand over distance; central system adaptation will be defined by the similarity in response between the exposed hand and the unexposed hand. Examining and comparing the forms of information used at the local level with that used at the central level should uncover important clues about the basic mechanisms governing the visual-proprioceptive representation of space. 


\section{Proposed Experiment}

To determine the kind of information that is used to encode a target's location, adaptation will be generated at an intermediate distance and there will be a test for after-effects at the same and at two novel distances, one closer and one farther. To assess the type of information that may be used in the central representation of target displacement and that is available for intermanual transfer, we will have the observer expose only the right hand and test both hands to measure the amount of transfer to the unexposed, left hand. The unexposed hand is isolated from any local changes in felt arm position in the exposed hand. The relative differences in pre-exposure and post-exposure pointing response across the testing distances will specify what type of information has been used in the process of adaptation for both the exposed and the unexposed hands. A comparison of post-pointing responses for the two hands will reveal the differences in adaptation when only visual information or when the combination of visual and proprioceptive information is available.

Adaptation has been generated if post-exposure responses are located above or below the target as a function of the target's direction of shift during exposure. Support for a radial model exists if post-exposure responses are located further from the target as the target's distance increases. In contrast, support for a linear model exists if post-exposure responses are unaffected by the target's distance. 


\section{Method}

\section{Observers}

Twenty-four male and female San Jose State students participated in the experiment to fulfill a course requirement of their General Psychology class. All observers were right-handed and had normal vision (at least 20/40) or vision corrected to normal using contact lenses. Those observers who used glasses participated only if they were able to see the target clearly without using their glasses (at the distances from 20 to $40 \mathrm{~cm}$ ).

\section{Apparatus}

A beam splitter (a half-silvered mirror) prevented the observer from seeing the testing hand when pointing to the virtual target in the dark (see Figure 1). The observer viewed the virtual target through a fair of goggles mounted to the column housing the beam splitter. The target was projected onto the mirror at each of the testing distances. The observer adjusted the stool to a proper height for looking through the goggles comfortably; excessive head movement was restricted by a chin rest. The chin rest was also used to position each observer's head so that the target would lie in the eye level plane (see Figure 1).

A KURTA digitizing tablet connected to a Macintosh personal computer registered and recorded the location of the responses using a Hypercard Stack. The Hypercard Stack gave the observer auditory feedback only when the pointing response was registered by the digitizing tablet. An electronic metronome, which sounded once every $5 \mathrm{~s}$, signaled the observer to point. 
Figure 1. The hand-eye coordination apparatus during a training phase with the target shifted downward (prism base up).

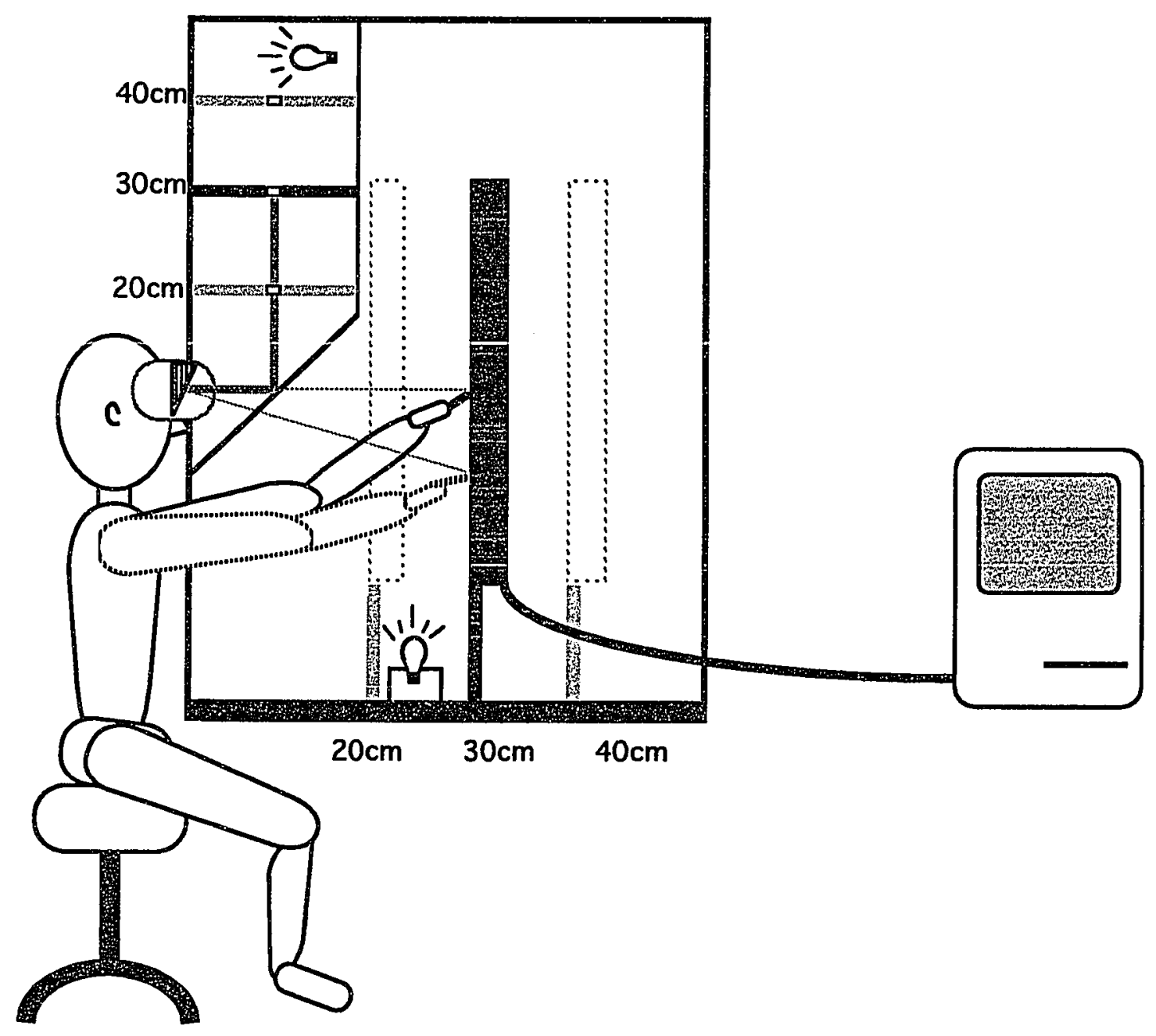




\section{Procedures}

Observers were randomly assigned to one of two training groups; each training group contained twelve observers. One group viewed a target shifted $11.3^{\circ}$ upward and the other group viewed a target shifted $11.3^{\circ}$ downward. The target's image was shifted by having the observer view the target through a set of $20^{\circ}$ prisms that was oriented either base up or base down.

All observers were tested with the target projected at each of three testing distances $(20 \mathrm{~cm}, 30 \mathrm{~cm}$, and $40 \mathrm{~cm})$. These distances were measured from the plane of the eyes to the front surface of the touchpad. When the three testing distances were balanced across the two training groups, a total of six experimental conditions was generated.

Observers completed a total of three experimental sessions, one for each of the three testing distances. Each session lasted approximately thirty minutes and was divided into two halves, an exposure sequence and a control sequence. During a 7-min break which separated the two sequences, observers performed a simple hand-eye coordination task; they played catch with the experimenter. Each session began with an exposure sequence and concluded with a control sequence. Each exposure sequence included three parts:

(1) Baseline phase, the observer pointed 20 times, alternating left and right hands, to the reflection of a target in otherwise complete darkness;

(2) Training phase, the observer pointed 40 times with the right hand to the reflection of a target viewed through a set of prisms; and 
(3) Testing phase, the observer, as in the Baseline phase, pointed 20 times, alternating left and right hands, to the reflection of a target in otherwise complete darkness.

The distance of the target during the Testing phase corresponded to that during the Baseline phase; for all Training phases, the target was presented only at $30 \mathrm{~cm}$.

For each trial, the observer pointed when the metronome signaled the start of a 5-s interval and returned the pointing hand to a specified resting position until the signal repeated. In the Baseline and the Testing phases, the observer exchanged pointing hands following each pointing response by passing the cursor pen from hand to hand. All phases began with the right hand.

Throughout both Baseline and Testing phases, observers did not see their hands; the pointing responses were made in darkness while viewing the reflection of a target. In the Training phase, when the pen contacted the touchpad a light flashed for less than $500 \mathrm{~ms}$ and revealed the position of the observer's hand and the pen in relation to the actual target location (terminal exposure). Observers were instructed to use this visual feedback to make error corrections on ensuing pointing responses.

After the exposure sequence, observers completed a control sequence that was identical to the exposure sequence except no prism was present during the training phase. This control sequence was included to minimize the residual after-effect (Welch et al., 1974) present when the observer returned for subsequent sessions. Each observer completed one of the three 
required experimental sessions per appointment, and appointments were separated by an interval of at least 24-hours.

\section{Data Scoring}

The pre-exposure and post-exposure pointing responses were measured as deviation $(\mathrm{cm})$ from the objective target. For both Target Position conditions, a positive score indicates that a response lies above the target; a negative score indicates that a response lies below the target. The mean negative after-effect is defined as the change in pointing response that results from the exposure to a discrepancy between the seen and the felt location of a target. This measure known as "adaptive shift " (Welch, 1974) was used to define the change in hand-eye coordination resulting from adaptation to a visually shifted target. It is calculated by subtracting preexposure pointing location (baseline) from post-exposure pointing location.

\section{Results}

Generally, the mean pre-exposure and post-exposure pointing responses were located below the actual target location (Table 1). The post-exposure values were closer to zero than the pre-exposure values; post-exposure values for both directions of the target shift were expected to be equal and average to zero. The absolute differences between pre- and post-exposure pointing responses were larger for the Exposed Hand; the Unexposed Hand did not show much change between pre- and post-exposure pointing after exposure to each of the two Target Position conditions (Table 2.).

Pre- and Post-exposure Pointing Responses

A 2 (Target Position) $\times 3$ (Testing Distance) $\times 2$ (Test Hand) $\times 2$ (Pre/Post-exposure Pointing ) $\times 10$ (Trials) mixed design 
Table 1.

Means and Standard Deviations for Pre-exposure and Post-exposure Pointing Responses Collapsed Across Conditions of Trials, Target Position and Test Hand.

\begin{tabular}{rccc}
\hline Pre/Post Exposure & Test Distance & Distance from Target $(\mathrm{cm})$ \\
\hline \hline \multirow{2}{*}{ Pre-exposure } & & Mean & S.E. \\
& & -2.26 & 0.14 \\
& $20 \mathrm{~cm}$ & -1.86 & 0.17 \\
& $30 \mathrm{~cm}$ & -1.77 & 0.18 \\
& $40 \mathrm{~cm}$ & & \\
Post-exposure & & & \\
& & -1.18 & 0.17 \\
& $20 \mathrm{~cm}$ & -1.56 & 0.21 \\
& $30 \mathrm{~cm}$ & -1.41 & 0.24 \\
\hline
\end{tabular}


Table 2.

Means and Standard Deviations for the Absolute Difference Between Preexposure and Post-exposure Pointing Responses Collapsed Across Target Position.

\begin{tabular}{rccc}
\hline Test Hand & Test Distance & Distance from Target $(\mathrm{cm})$ \\
\hline Exposed & & Mean & S.E. \\
& $20 \mathrm{~cm}$ & 2.35 & 0.39 \\
& $30 \mathrm{~cm}$ & 2.91 & 0.40 \\
& $40 \mathrm{~cm}$ & 3.75 & 0.38 \\
& & & \\
Unexposed & & 1.69 & 0.34 \\
& $20 \mathrm{~cm}$ & 1.90 & 0.41 \\
& $30 \mathrm{~cm}$ & 2.20 & 0.33 \\
\hline
\end{tabular}


analysis of variance (ANOVA) was performed on all pointing responses. The direction of Target Position (prism base orientation) was the between-subjects variable in the analysis. This analysis was conducted to determine if there were significant differences between pre- and post-exposure pointing responses across Trials. Also assessed was a systematic drop in all responses relative to the target from trial to trial; this may be a possible artifact in the data. The pre- and post-exposure responses for both Exposed and Unexposed hands across Trials are presented graphically in Figures 2.

There was a significant main effect of Trials $[\mathrm{E}(9,198)=39.86, \mathrm{p}<.01]$. For the Exposed and Unexposed hands both before and after exposure, observers pointed lower on successive trials with respect to the target. This artifact can be described as a drift in a downward direction relative to the target of all preand post-exposure pointing responses. A main effect of Pre-/Post-exposure was not found $[\mathrm{F}(1,22)=1.27$, n.s. $\mathrm{p}<.28]$ but, Trials interacted independently with Pre-/Post-exposure and with Test Hand in the analysis. Two two-way interactions of Trials X Pre-/Post-exposure $[\mathrm{F}(9,198)=5.12, \mathrm{p}<.01]$ and Trials $X$ Test Hand $[\mathrm{F}(9,198)=5.96, \mathrm{p}<.01]$ were significant.

A comparison of Trials was significant; the first half (trials 1-5) of the ten responses were different from the last half (trials 6-10) $[\mathrm{E}(1,396)=28.46$, $\mathrm{p}<.01]$. Because there was a main effect of Trials and two-way interactions of Trials $X$ Test Hand and Trials $X$ Pre-/Post-exposure, a follow-up analysis was needed to interpret the effects of exposure.

Although there was instability in the pre-exposure responses in trials 1-5 this same pattern existed in the post-exposure responses of trials 1-5. In trials 6-10, the downward drift did not exist, and any effect due to exposure 
Figure 2.

The Effect of Trials on Pre- and Post-exposure Pointing Responses Averaged Over Test Hand, Test Distance, and Target Position.

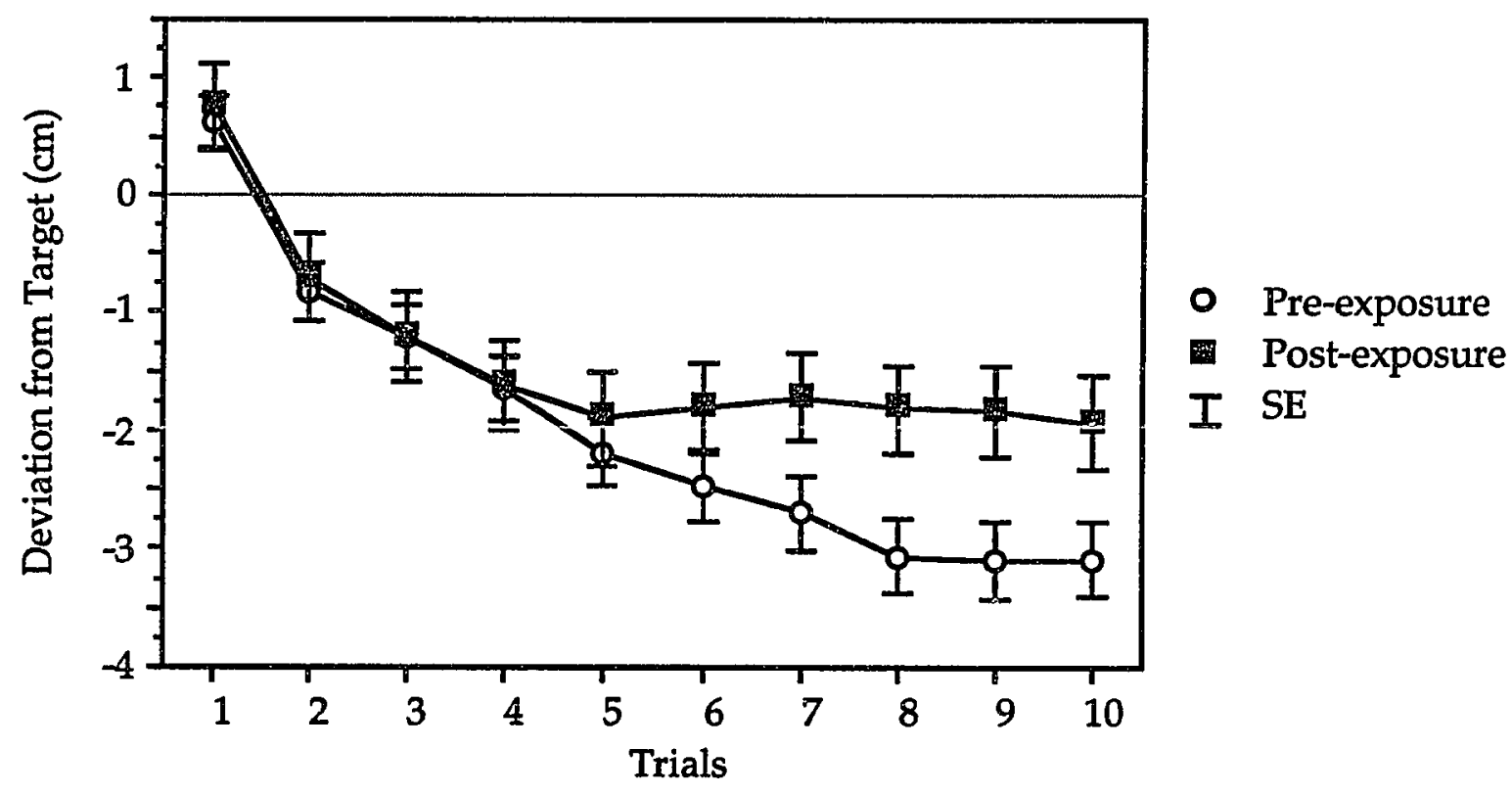


had been washed out when the arm drop had reached asymptote. For this reason, only trials 1-5 were included in the analysis; pointing responses for the first half (trials 1-5) of the ten responses were averaged and entered into an analysis of variance. The analysis examined the change in pointing responses between the two hands before and after viewing the target through the prisms; trials 1-5 were used after recoding pre- and post-exposure pointing responses into adaptive shift.

\section{Adaptive Shift Trials 1-5}

A 2 (Target Position) $\times 2$ (Test Hand) $\times 3$ (Testing Distance) mixed design analysis of variance (ANOVA) was performed on pointing responses collapsed across Trials and recoded as mean adaptive shift. The direction of Target Position (prism base orientation) was the between-subjects variable in the analysis. The adaptive shift values were coded as absolute difference between pre-exposure and post-exposure responses and should be interpreted as the change in pointing response after exposure; negative adaptive shift values indicate lack of adaptation.

A significant main effect of Test Hand was found $[\mathrm{E}(1,22)=34.21, \mathrm{p}<.01]$. This difference in mean location of post-exposure pointing response relative to the pre-exposure responses supports the hypothesis that local adaptation due to exposure to the optically-shifted target was generated. Therefore, adaptive shifts for the Exposed hand were larger than those for the Unexposed Hand.

The transfer of adaptive shift ranged from $79 \%$ to $47 \%$ as Test Distance increased (see Table 3); the adaptive shifts were no different across distance $[\underline{F}(2,44)=.63$, n.s. $\mathrm{p}<.54]$. This result does not show support for complete 
intermanual transfer from the Exposed to the Unexposed Hand. Additionally, the shows support for a transfer of linear information (a constant correction) at the central level of spatial mapping.

The main effect of Test Distance was not significant $[F(2,44)=2.29$, n.s. $p>.11]$ due to a two-way interaction of Test Hand $X$ Test Distance $[E(2,44)=4.89$, $\mathrm{p}<.01]$. The adaptive shift of the Exposed Hand increased as a function of increased Test Distance ranging from 2.27 to $3.92 \mathrm{~cm}$ (see Table 3); this relationship was linear $[\mathrm{F}(1,44)=11.54, \mathrm{p}<.01]$ (see Figure 3). This finding supports the proposed radial model of proprioceptive mapping.

Discussion

The results from this study indicated that 1) there was a change in pointing responses for the Exposed Hand after exposure that was not present in responses for Unexposed Hand, and 2) this change was related to the distance at which the target was presented. Exposed vs Unexposed

Observers pointed their Unexposed Hand to similar locations both before and after exposure while they pointed their Exposed Hand to locations which were above or below the target depending on which Target Position they had during exposure. A larger degree of adaptation was generated when the combination of proprioceptive and visual information was available to the Exposed Hand than when only visual information was available to the Unexposed Hand. This finding supports both the notion that adaptation results from a change in the felt position of the Exposed arm (Harris, 1963; 1968; Templeton et al., 1974) or that there is a change in the relationship between the seen and felt location of the target (Held \& Freedman, 1963). 
Table 3. Mean Adaptive Shift for Trials 1-5 for the Exposed and Unexposed

Hands Over the Three Testing Differences Collapsed Across Trials 1-5 and Direction of Target Shift.

\begin{tabular}{cccc}
\hline Test Hand & Test Distance & \multicolumn{2}{c}{ Adaptive Shift } \\
\hline \hline \multirow{2}{*}{ Exposed } & & Mean & S.E. \\
& & 2.27 & 0.32 \\
& $20 \mathrm{~cm}$ & 2.93 & 0.45 \\
& $30 \mathrm{~cm}$ & 3.98 & 0.37 \\
& $40 \mathrm{~cm}$ & & \\
& & & \\
Unexposed & & 1.79 & 0.41 \\
& $20 \mathrm{~cm}$ & 2.21 & 0.41 \\
& $30 \mathrm{~cm}$ & 1.86 & 0.33
\end{tabular}


Figure 3.

Linear Relationship Between Adaptive Shift and Test Distance for the Exposed and Unexposed Hand.

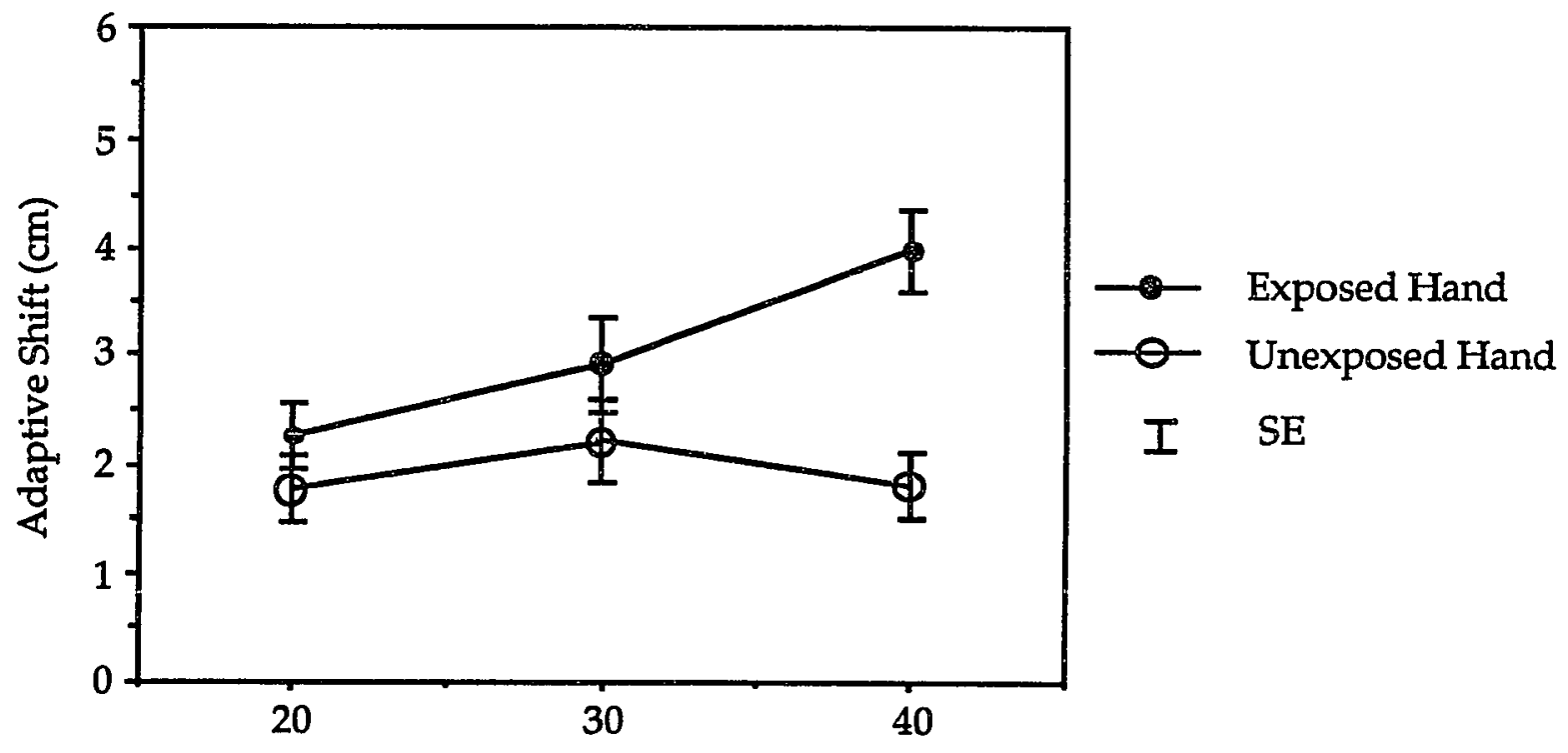

Test Distance $(\mathrm{cm})$ 


\section{Intermanual Transfer}

There was an adaptive shift found in the Unexposed hand and, like Cohen (1967) who found intermanual transfer in adaptation to laterally displacing prisms, there was transfer from the Exposed to Unexposed hand. Cohen (1967) hypothesized that the change in the judgment of gaze direction was the information that accounted for the adaptive shift of post-exposure responses he observed in the Unexposed hand. By restricting head movement, the only direct proprioceptive information available to guide the Unexposed hand was from the ocular muscles signaling the change in gaze direction. Contrary to this angular encoding using the direction of gaze proposed by Cohen (1967), the results of the present study suggests that a learned linear compensation is transferred at the central level.

The transfer of a linear compensation to the Unexposed Hand suggests that a combination of both proprioceptive and visual information available at the local level is required to accurately map object locations within reach or extrapersonal space. The isolation of the Unexposed hand from local proprioceptive information in the Exposed hand defines a primary source of adaptation as the change in local proprioceptive mapping due to exposure. The change in the felt position of the Exposed arm (Harris, 1963; 1968) and the notion that linkages within the general proprioceptive map (Templeton et al., 1974; Wallace \& Redding, 1992b) are strongly supported by this finding.

For the purposes of this study, the proprioceptive and visual representation systems are assumed to be both central and local representation systems. The present experiment addresses the type of information used in adaptation to a visually displaced object and it is not 
meant to be a strong test of visual versus proprioceptive mapping at either the local or central level. It was assumed that only central proprioceptive and visual information from the Exposed "adapted" hand was available to the Unexposed hand.

Radial Model

Adaptive shift increased as a function of increased Test Distance for the Exposed hand. There was a linear relationship between increased adaptive shift and increased Testing Distance, suggesting that the observer may have encoded location in the Exposed hand along a radial reference from a point along the body. If the adaptive shift values are transformed into angular measures, a constant angular displacement is defined. This angular displacement can be represented by the eye as a line of sight; since the eye rotates in a socket, the afferent information it receives from its musculature is coded as an angular deflection. If parsimony is the rule, observers encoded the location of a visually-shifted target in visual/proprioceptive space along a radial which was referenced with respect to the line of sight that a target occupies.

If the observer truly conserves angular information, all reaching trajectories to targets which lie on a particular radial should have the same angular position regardless of a target's distance. Therefore, a strong test of this theory should require the recording and comparison of hand trajectories in a visually open-loop pointing task while using the identical testing paradigm. This would be a primary test in an attempt to discover the type of information used in mapping three-dimensional space. 


\section{Further Research}

A priori, it might have been anticipated that the exposed arm would experience fatigue after reaching out to a target 60 times. Since the Unexposed hand was required to point only a total of 20 times, post-exposure responses from this hand would not be expected to drift downward over the same time course and/or magnitude as the exposed arm. The general downward drifting of all pointing responses, both baseline and post-exposure sequences, indicates that fatigue is not the primary cause of the drift. Can this instead be due to a unstable or fragile representation of spatial location for hand-eye coordination in the vertical plane?

It seems that proprioceptive information breaks down quickly when visual information is absent. In the vertical dimension, maintaining the spatial mapping of a location would include the relationship between force and tension in the activated muscles because force is required to lift the arm against gravity. If adaptation was focused in the proprioceptive component for the observers, it was disrupted as they repeatedly lifted their hands. In normal circumstances, where visual feedback is available, any problems with accuracy are quickly resolved by continuous recalibration of the spatial map.

The majority of the literature examines adaptation in the lateral or horizontal dimension (Cohen, 1967; Harris, 1963; 1968; Templeton et al., 1974; Held \& Freedman, 1963). The cues for location in the lateral dimension are many, and the body midline is certainly one compelling cue. Another more useful cue is the relationship among the paired muscle groups 
(agonist/antagonist) of the body whose purposes are to move or position limbs in given direction.

It would be unwise to assume that the same conditions apply for adaptation to a visually shifted target in the vertical and the lateral dimensions of proprioceptive space. At any given articulation, muscles are activated in opposition to one another, the ratio of force and tension in this muscle pairing creates a mapping of the angular position or movement of the limb. In the vertical dimension, an additional force in the relationship that must be accounted for by this proprioceptive map is gravity.

A simple way to eliminate the confounding effects of gravity would be to rotate the testing paradigm 90 degrees on its side. Gravitational information would be held constant for the direction of optical shift by having it aligned orthogonally to the plane of the adaptation. The importance of visual and proprioceptive information could be assessed for the vertical plane of the body removed from gravity. Direct comparisons may then be made to lateral displacement studies.

Another way to asses the possible role that gravitational information plays in the proprioceptive mapping would be to submerge the observer in water and use the same paradigm. When submerged, buoyancy will counteract the weight of the limbs so that all visual information would theoretically remain intact while the additional force of gravity would be removed from proprioceptive information. By comparing these proposed experiments with the results of the present study, advances would be made in understanding what types of information are used to map visual and proprioceptive space. 


\section{References}

Cohen, M. M. (1967). Continuous verses terminal feedback in prism aftereffects. Perceptual Motor Skills, 24, 700-705.

Cohen, M. M. (1973). Visual feedback, distribution of practice, and intermanual transfer of prism aftereffects. Perceptual Motor Skills, $\underline{38}$, 599-609.

Harris, C. S., (1963). Adaptation to displaced vision: visual, motor, or proprioceptive change? Science, 140, 812-813.

Harris, C. S., (1968). Perceptual adaptation to inverted reversed, and displaced vision. In R. N. Haber (Ed.), Contemporary theory in visual perception (pp. 504-528). New York, NY: Holt, Rinehart \& Winston Inc.

Hay, J. C. \& Pick, H. L. Jr. (1966). Visual and proprioceptive adaptation to optical displacement of the visual stimulus. Journal of Experimental Psychology, 71, 150-158.

Held, R. \& Freedman, S. J. (1963). Plasticity in human sensorimotor control. Science, 142, 455-462.

Jeannerod, M. (1988) The neural and behavioral organization of goal-directed movement . Oxford, Britain: Oxford University Press.

Jeannerod, M. (1983). How do we direct our actions in space? In A. Hein \& M. Jeannerod (Eds.), Spatially oriented behavior ( pp. 1-13). New York, NY: Springer-Verlag.

Jeannerod, M. (1991). A neuropsychological model for the directional coding of reaching movements. In J. Paillard (Ed.), Brain and space (pp. 49-69). Oxford, Britain: Oxford University Press. 
Osgood, C. E. (1949). The similarity paradox in human learning: A resolution. Psychological Review, 56, 132-143.

Redding, G. M. \& Wallace, B. (1988). Adaptive mechanisms in perceptualmotor coordination: Components of prism adaptation. Journal of Motor Behavior, 20, 242-254.

Redding, G. M. \& Wallace, B. (1992a). Effect of pointing rate and availability of visual feedback on visual and proprioceptive components of prism adaptation. Journal of Motor Behavior, 24, 226-237.

Redding, G. M. \& Wallace, B. (1992b). Adaptive eye-hand coordination: Implications of prism adaptation for perceptual-motor organization In L. Proteau \& D. Elliot (Eds.)Vision and Motor Control (pp. 105-128). Amsterdam, Netherlands: North-Holland.

Rock, I. (1966). The Nature of Perceptual Adaptation. New York, NY: Basic Books Inc.

Schmidt, R. A. (1988). Motor Control and Learning: A Behavioral Emphasis 2nd ed. Champaign, IL: Human Kinetics Pub. Inc.

Templeton, W. B., Howard, I. P., \& Wilkinson, D. A. (1974). Additivity of components of prismatic adaptation. Perception and Psychophysics. $15,249-257$.

Welch, R. B., Choe, C. S., \& Heinrich, D. R. (1974). Evidence for a threecomponent model of prism adaptation. Journal of Experimental Psychology, 103, 700-705.

Welch, R. B. (1978). Perceptual Modifications. New York, NY: Academic Press. 
Welch, R. B. \& Cohen, M. M. (1991). Adapting to prismatic displacement. In S. R. Ellis, M. K. Kaiser, \& A. J. Grundwald (Eds.)Pictoral Communication in Virtual and Real Environments (pp. 295-304). Washington DC: Taylor \& Francis. 